\title{
Generation of gold nanoparticles according to procedures described in the eighteenth century
}

\author{
Álvaro Mayoral • Javier Agúndez • \\ Ignacio Miguel Pascual-Valderrama • Joaquín Pérez-Pariente
}

Published online: 20 April 2014

(C) The Author(s) 2014. This article is published with open access at Springerlink.com

\begin{abstract}
Gold nanoparticles have received much attention in recent years due to their unique size-dependent properties, as they find useful applications in materials science [Mayoral et al. (Nanoscale 2:335-342, 2010)], catalysis [Schwerdtfeger (Angew Chem Int Ed 42:1892-1895, 2003)] [Hashmi and Hutchings (Angew Chem Int Ed 45:7896-7936, 2006)] and biology [Sperling et al. (Chem Soc Rev 37:1896-1908, 2008)]. The preparation of such nanoparticles benefits from modern chemical knowledge, and a large variety of several procedures have been developed aiming at controlling the size and shape of these metal nanoparticles. Here, we show that two eighteenth-century recipes (Online Resource 1) used at that time to prepare drinkable solutions of gold, used as drugs, actually generate gold nanoparticles, clusters and even monoatomic species of gold. These simple methods involve the dissolution of gold in a solution of ammonium chloride in nitric acid (aqua regia) and the mixing of the resulting solution with rosemary or cinnamon essential oils. The complex mixture of compounds resulting from the fast reaction between aqua regia and the essential oils behave simultaneously as reductants and stabilisers of the nascent gold particles. These results not only prove that historical speculations on
\end{abstract}

Electronic supplementary material The online version of this article (doi:10.1007/s13404-014-0139-8) contains supplementary material, which is available to authorized users.

\section{Á. Mayoral}

Laboratorio de Microscopías Avanzadas,

Instituto de Nanociencia de Aragón, Universidad de Zaragoza,

Mariano Esquillor, Edificio I+D, 50018 Zaragoza, Spain

J. Agúndez $\cdot$ J. Pérez-Pariente $(\bowtie)$

Instituto de Catálisis y Petroleoquímica,

Marie Curie, 2, Cantoblanco 28049, Madrid, Spain

e-mail: jperez@icp.csic.es

I. M. Pascual-Valderrama

I. E. S. Lope de Vega, San Bernardo, 70, Madrid, Spain the presence of finely divided gold particles floating in these solutions were basically correct but they could also serve as a source of inspiration for new experimental approaches procuring the generation of stable sub-nanometer gold nanoparticles.

Keywords Gold nanoparticles · STEM · Eighteenth-century recipes $\cdot$ Essential oil

There is a general agreement that the first scientific approach to the nature of what are now known as solutions of colloidal gold can be traced back to the works of the British scientist Michael Faraday. In a seminal paper published in 1857 [1,2], he clearly concluded that the ruby or purple colours exhibited by solutions containing gold were due to the presence of very small particles of this metal, so small that they were invisible to the human eye, yet they could be easily detected by their effect in dispersing incident light.

As important as this publication is as pioneering the foundation of modern colloid chemistry, Faraday was not the first in preparing such ruby-coloured solutions neither in suggesting the presence in them of gold in such an extremely divided state.

There is a long tradition behind the preparation of colloidal gold, which can be briefly summarised as follows. Historically, two main research lines were developed aiming at two different purposes. One of them was directed toward the preparation of colloidal gold to be used for colouring the transparent glass into red colour, resulting in the much sought, valuable and beautiful ruby glass [3]. The second approach belongs to the field of medicine, where the goal was to prepare a gold drinkable solution which behaved as a powerful drug able to cure even the most severe ailments, the so-called potable gold, known in old Latin texts as aurum potabile [4, 5]. Although its origin can be traced back to the Middle Ages, 
the preparation of potable gold was still a subject of interest in the eighteenth century.

The editor and commentator of the 1757 edition of the French chemist and pharmacist Nicholas Lemery's Course of Chemistry, M. Baron, included in this edition a recipe that described the preparation of the so-called potable gold of Mademoiselle Grimaldi [6]. Few years later, the French chemist Pierre-Joseph Macquer commented extensively on the recipe in his Dictionary of Chemistry (1766) [7]. He argued that the red solution resulting from the Grimaldi's procedure contains elemental gold in a state of extreme division, and the particles were "floating in the oily fluid" [8].

Would it be possible that the Grimaldi's recipe actually generates colloidal gold, i.e., gold nanoparticles and/or clusters? Could the conclusion of Macquer be experimentally corroborated, anticipating in this way for one century the Faradays' view? The Grimaldi's procedure is described with enough detail as to be suitable for replication in a modern laboratory, and moreover, as we will see, it involves the use of natural products, plant-derived organic substances, which would make it particularly appealing in the view of decreasing the input of synthetic molecules, which in many cases come from more complicated reactions involving different chemicals and producing a large amount of unwanted side products, in the growing field of eco-friendly nanotechnology.

The procedure described in the Lemery's treatise starts by dissolving gold in aqua regia. Then, the resulting goldenyellow solution is mixed with rosemary essential oil, and the mixture is let to stand for a while, in such a way that two layers are formed. The aqueous lower layer loses rapidly its colour, while the upper one is coloured in yellow. From this observation, the author of the recipe concluded that the gold was being taken up from the aqueous phase to the supernatant rosemary oil phase. Then, a portion of this oily layer is dissolved in alcohol (ethanol), which results in a beautiful red solution, which is the Grimaldi's potable gold, the solution that Macquer supposed shall contain gold in a state of extreme division.

We have replicated this procedure by using the same proportion of the recommend reagents, assuming that the aqua regia was prepared by mixing concentrated nitric acid and ammonium chloride (see "Experimental" section). By following this procedure, a dark-brown supernatant solution is immediately obtained after mixing the rosemary oil and the gold solution placed in a separating funnel (Online Resource 2). Twenty-four hours later, golden particles are observed in the interphase between the two layers, the upper oil and the lower aqueous phases, while the yellow colour of the aqueous phase is much less intense (Online Resource 3). This behaviour suggests that the gold has been driven from the aqueous to the oily phase, but at least part of it is reduced again to the metal state and concentrates just at the interphase.
The blackening of the oily phase is not described in the 1757 recipe. We then decided to let the mixture stand still to observe the evolution of the system. The oily layer evolved with time; and after 15 days, its colour was dark red, while that of the aqueous lower layer was pale yellow. At this point, we took an aliquot of the oily red phase, which was readily dissolved in absolute ethanol, while the aqueous phase was also sampled to analyse the presence of gold in there. Chemical analysis revealed that more than $99 \%$ of the gold initially present in the acid aqueous phase had been removed, which was in agreement with the observation described in the old recipe. Moreover, the transparent ethanol solution exhibited an orange-red colour with a concentration of gold of $220 \mathrm{mg} / \mathrm{L}$. (Online Resource 4). The replication of the Grimaldi's recipe reported here is in this way more complete and different than what Vanino reported in 1906, where he used rosemary oil as a reducing agent of a diluted solution of gold chloride in water without the intervention of aqua regia [9].

Traditionally, the analysis and therefore the observation of the nanoparticles presumably formed was an extremely complicated task due to the low resolution of the available transmission electron microscopes. Nowadays, with the implementation of the spherical aberration $\left(\mathrm{C}_{\mathrm{s}}\right)$ correctors $[10,11]$, subangstrom resolution can be commonly attainable allowing imaging small clusters or even single atoms [12]. For the current analysis, we used an aberration-corrected scanning transmission electron microscope (STEM), which is the most appropriate technique for imaging heavy elements as gold. A drop of the as-synthesised ethanol solution was placed onto a holey carbon copper microgrid, which was subsequently let to dry. Further details can be found in the "Experimental" section.

Two main kinds of gold species were observed in the solution. Gold nanoparticles of an average size of $5 \mathrm{~nm}$, being the smallest one of $2 \mathrm{~nm}$ and the largest $19 \mathrm{~nm}$, but $90 \%$ fall in the range of 2 to $8 \mathrm{~nm}$, see Fig. 1. The most typical type of nanoparticles formed ( 3 to $4 \mathrm{~nm}$ ) mainly adopted decahedral

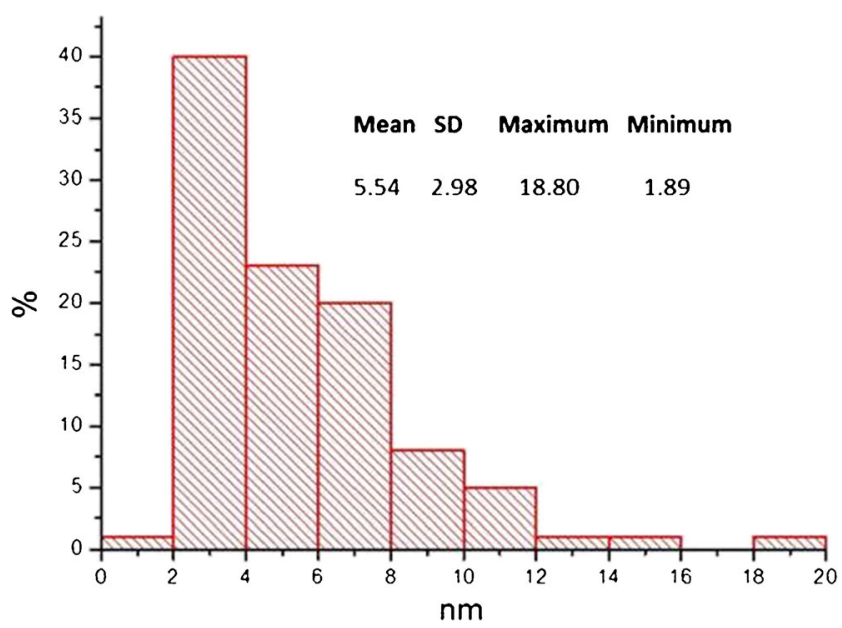

Fig. 1 Histogram of gold nanoparticle size distribution 
Fig. 2 Cs-corrected STEMHAADF images of three different types of Au nanoparticles a decahedra, b icosahedra and $\mathbf{c} f c c$ symmetry with the correspondent FFT diffractograms at the bottom
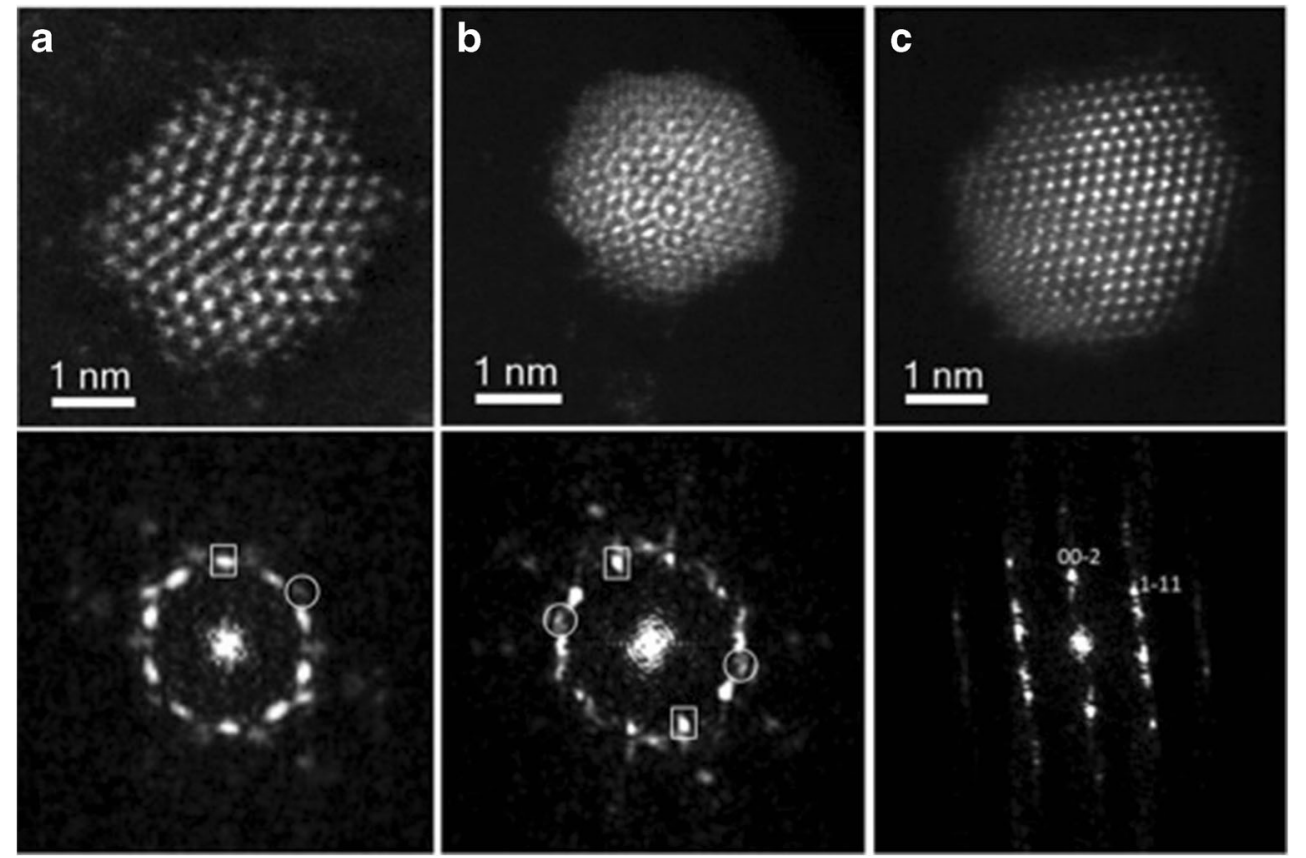

or icosahedral morphology, while $f c c$-twinned nanoparticles [13] are less abundant. Figure 2a displays a $2.7 \mathrm{~nm} \mathrm{Au}$ decahedron on its 5-fold axes displaying two main crystallographic distances of 2.37 and $2.07 \AA$ corresponding to the (111) and (200) planes, respectively. The fast Fourier transform (FFT) corroborating the 5-fold symmetry is shown at the bottom. Figure $2 \mathrm{~b}$ corresponds to a $3.45 \mathrm{~nm} \mathrm{Au}$ icosahedron also displaying its 5-fold symmetry axes, both FFTs exhibits the (111) and (200) reflections, marked with rectangles and circles, respectively. Figure $2 \mathrm{c}$ corresponds to a twinned $f c c$ $4.2 \mathrm{~nm}$ nanoparticle along the [110] orientation. The second clearly differenced type of gold species observed correspond to small disordered clusters of about 1-1.5 nm, which also appeared together with isolated gold atoms (Fig. 3).

The recipe reported in the Lemery's treatise is not the only one described in academic texts in the eighteenth century on the preparation of gold solutions obtained by means of essential oils. The German physician F. Hoffmann reported in 1722 [14] the preparation of a "gold tincture" by mixing a solution of gold in aqua regia with a mixture of cinnamon essential oil and ethanol under gentle heating. A dark-brown, resin-like product is obtained, from which a supernatant solution can be separated (Online Resource 5). The examination of this product by TEM reveals the presence of gold nanoparticles that reached sizes up to $20 \mathrm{~nm}$. Different structural conformations of gold could be observed, which varied from multi-twinned crystals (Fig. $4 \mathrm{a}$ ), single crystal $f c c$ nanoparticles, icosahedra (Fig.4b, c) and small clusters of few atoms surrounded by isolated gold atomic species (Fig. 4d).

These two procedures render quite similar results and confirm the Macquer's hypothesis regarding the presence of extremely divided gold.

It is noticeable the presence in these solutions of very small clusters of gold and even isolated atoms of this metal, which points out to a remarkable role of the oily phase resulting from the fast chemical reaction between the aqua regia and the essential oils in preventing the overgrowth of the primary gold particles into larger aggregates. In this way, the oily phase behaves at the same time as a reducing and a very efficient capping agent [15-17], leading to a simple method to prepare
Fig. $3 \mathrm{C}_{\mathrm{s}}$-corrected STEM-

HAADF images of a several $\mathrm{Au}$ clusters, $\mathbf{b}$ a magnified image of a 1-nm Au cluster and $\mathbf{c} \mathrm{Au}$ cluster together with several isolated $\mathrm{Au}$ atoms
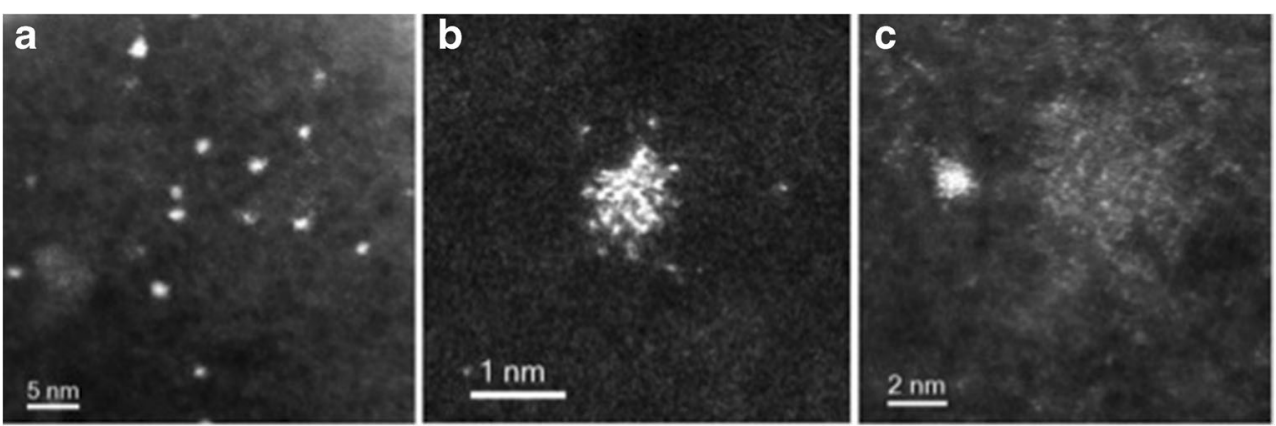


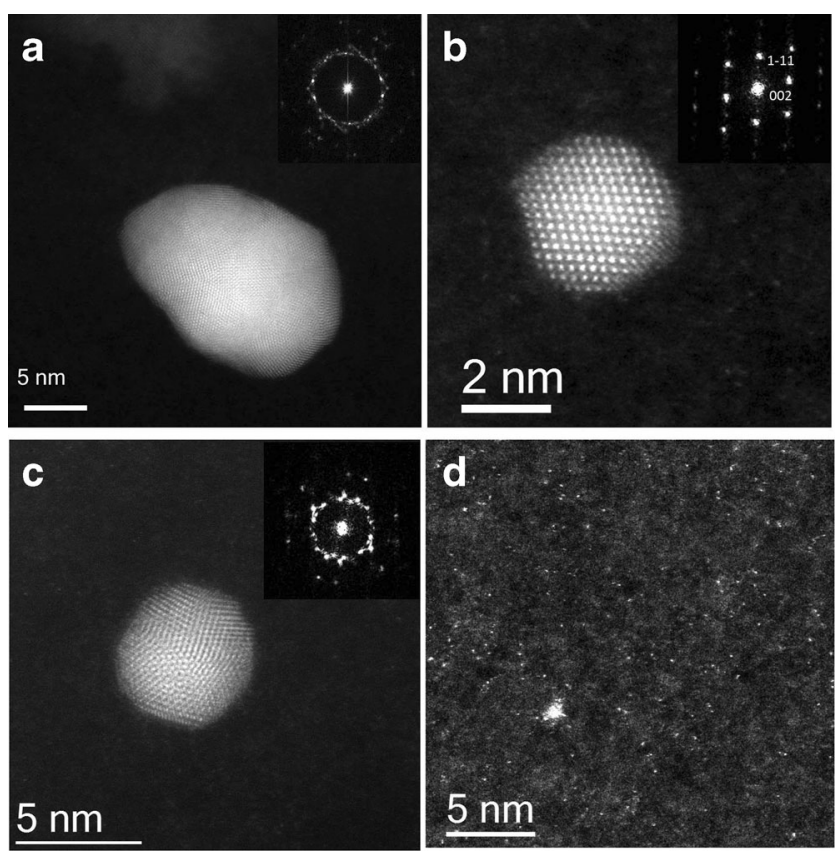

Fig. $4 \mathrm{C}_{\mathrm{s}}$-corrected STEM-HAADF images of a multi-twinned crystal, b-c single crystal $f c c$ nanoparticles and icosahedra and d small Au clusters of few atoms surrounded by isolated gold atomic species

gold nanoparticles constituted by a very small number of atoms and even single-atom species. This effect of the essential oils shall be related to their specific chemical composition and the way their individual chemical components react with the aqua regia to yield complex mixtures of oxidation compounds. In this regard, it is worth mentioning that the rosemary essential oil used in this work contains a mixture of terpenic hydrocarbons dominated by alpha-pinene and camphene and the oxygen-containing terpenes 1,8-cineole and camphor, while the major component of the cinnamon essential oil is eugenol (see "Experimental" section).

This aspect would be worth of further investigation, for it could inspire new experimental approaches toward the generation of stable sub-nanometer gold nanoparticles, which have been reported to be extremely active in the oxidation of organic substrates with oxygen [18].

\section{Experimental section}

Replication of the Hoffmann's recipe

A gold nugget $(0.154 \mathrm{~g})$ was dissolved in $18.5 \mathrm{~g}$ of aqua regia $\left(\mathrm{NH}_{4} \mathrm{Cl} / \mathrm{HNO}_{3} ; 1: 4\right.$ by mass; $\left.\mathrm{HNO}_{3}, 65 \mathrm{wt} \%\right)$. The mixture was stirred in a $50-\mathrm{mL}$ glass beaker placed in a sand bath at $55^{\circ} \mathrm{C}$. A solution of essential cinnamon oil in ethanol (1:3 by mass) was added (essential oil solution/Au solution 3:1 by mass). Some bubbles were immediately produced causing violent jets of liquid shooting the walls of the beaker, while the brown colour of the mixture becomes darker. In a few minutes, it evolved to a black viscose substance, as described in the original recipe, although careful examination revealed that it was in fact brown-reddish. The solution was cooled down to room temperature, and ethanol was added to dissolve a portion of the dense substance ( $2: 1$ by weight). The mixture was separated by decantation, and the supernatant liquid was observed under the transmission electron microscope to search for gold nanoparticles in it.

\section{Replication of the Lemery's recipe}

A gold nugget $(0.470 \mathrm{~g})$ was dissolved in $15.0 \mathrm{~g}$ of aqua regia $\left(\mathrm{NH}_{4} \mathrm{Cl} / \mathrm{HNO}_{3} ; 1: 4\right.$ by mass; $\left.\mathrm{HNO}_{3}, 65 \mathrm{wt} \%\right)$. The resulting golden solution was placed in a separating funnel, to which $7.5 \mathrm{~g}$ of rosemary essential oil were added. The oil became immediately dark brown by mixing. The system is let to stand for 15 days; and after that, the oily phase gets dark red. Then, a portion of the resulting red supernatant solution is taken and mixed with ethanol $(1: 5, w / w)$. This solution is examined by TEM according to the method described in the paper.

\section{TEM images}

Electron microscopy was performed in a spherical aberration $\left(\mathrm{C}_{\mathrm{S}}\right)$-corrected transmission electron microscopy, Titan $\mathrm{X}$ FEG FEI $60-300 \mathrm{kV}$ (operated at $300 \mathrm{kV}$ ) equipped with a monochromator and a Gatan $2 \mathrm{k} \times 2 \mathrm{k}$ CCD camera. In addition to the STEM unit, the microscope is also fitted with a $\mathrm{CEOS}_{\mathrm{s}}$ corrector for the electron probe capable of achieving a resolution of $0.8 \AA$. For analytical measurements, the microscope is equipped with a Gatan Energy Filter Tridiem 866 ERS and an EDS detector.

\section{GC-MS analysis of essential oils}

The essential oils (rosemary and cinnamon) were analysed by gas chromatography-mass spectrometry (GC-MS) employing a gas chromatograph (Agilent 6890) coupled with a mass spectrometer (Agilent $5973 \mathrm{~N}$ ) using a capillary column made of methylpolysiloxane $(30 \mathrm{~m} \times 0.25 \mathrm{~mm} \times 0.25 \mu \mathrm{m})$, heating from 70 to $290{ }^{\circ} \mathrm{C}$ at $6{ }^{\circ} \mathrm{C} / \mathrm{min}$. The composition (wt $\%$ ) of the rosemary oil was $24.9 \%$ 1,8-cineole, $21.9 \%$ alpha-pinene, $20.91 \%$ camphor, $9.06 \%$ camphene, $3.81 \%$ borneol, $3.34 \%$ verbenone, $2.59 \%$ myrcene, $2.41 \%$ beta-pinene, $2.01 \%$ caryophyllene, $2.00 \%$ p-cymene, $1.16 \%$ alpha-humulene, $0.98 \%$ bornyl acetate, $0.94 \%$ gamma-terpinene, $0.63 \% 4-$ terpineol, $0.63 \%$ alpha-terpineol, $0.29 \%$ alpha-terpinolene, and $0.28 \%$ fenchone. The composition (wt $\%$ ) of the cinnamon oil was $87.96 \%$ eugenol, $3.14 \%$ caryophyllene, $1.13 \%$ alpha-pinene, $0.76 \%$ alpha-cubebene, $0.35 \%$ a-humulene, $0.26 \%$ beta-pinene, $0.24 \%$ linalool, $0.20 \%$ p-cymene, $0.13 \%$ methylphenyl benzoate, and $0.12 \%$ safrol. 
Acknowledgements The research leading to these results has received funding from the European Union Seventh Framework Programme under Grant Agreement 312483-ESTEEM2 (Integrated Infrastructure InitiativeI3). The Zentralbibliothek Zürich (service of Handschriften, Musik und Alte Drucke) is acknowledged for giving us access to the first edition of the book Observationum Physico-chymicarum selectiorum libri III, Haal, 1722, by F. Hoffman, and Dr. Ana B. Pinar is acknowledged for obtaining copies of the Observatio XIII and Observatio XXI of this book. We are also grateful to Dr. Hereward Tilton (Department of History, University of Exeter) for providing us with copies of some of the references. Prof. V. Fernández Herrero is acknowledged for his assistance in translating into English some of the cited references that are written in German. We are grateful to Dr. A. Cristina Soria Monzón and Prof. Jesús Sanz (Institute of Organic Chemistry, CSIC) for the GC-MS analyses of the essential oils. The help of Prof. Ermias Dagne (Dept of Chemistry, Addis Ababa University) in preliminary analyses of the oils is also acknowledged. Dr. L. Gómez-Hortigüela is acknowledged for the pictures of the rosemary oil experiment included in the extended data.

Open Access This article is distributed under the terms of the Creative Commons Attribution License which permits any use, distribution, and reproduction in any medium, provided the original author(s) and the source are credited.

\section{References}

1. Faraday M (1857) The Bakerian Lecture: experimental relations of gold (and other metals) to light. Philos Trans R Soc Lond 147:145-181

2. Tweney RD (2006) Discovering discovery: how Faraday found the first metallic colloid. Perspect Sci 14:97-121

3. Hunt LB (1976) The true story of Purple of Casius. Gold Bull 9:134 139

4. Hauser EA (1952) Aurum potabile. J Chem Educ 29:456-458
5. Highby GJ (1982) Gold in medicine. Gold Bull 15:130-140

6. Lemery N (1757) Cours de chymie, Paris.

7. Macquer PJ (1766) Dictionnaire de chymie. Paris, pp 174-177

8. Svedberg T (1921) The formation of colloids. J. and A. Churchill, London, $\mathrm{p} 74$

9. Vanino L, Hartl F (1906) Ueber die Bildung colloïdaler Goldlösungen mittels ätherischer Oele. Chem Ber 39:1696-1700

10. Krivanek OL, Delby N, Lupini AR (1999) Proceedings of the International Workshop towards Atomic Resolution Analysis-Port Ludlow, Washington, USA, 6-11 September 1998-Part 1: Techniques and instrumentation-Foreword. Ultramicroscopy 78: $1-11$

11. Haider M, Uhlemann S, Zach J (2000) Upper limits for the residual aberrations of a high-resolution aberration-corrected STEM. Ultramicroscopy 81:163-175

12. Mayoral A, Blom DA, Mariscal MM, Guiterrez-Wing C, Aspiazu J, Jose-Yacaman M (2010) Gold clusters showing pentagonal atomic arrays revealed by aberration-corrected scanning transmission electron microscopy. Chem Commun 46:8758-8760

13. Mayoral A, Barron H, Estrada-Salas R, Vazquez-Duran A, JoseYacaman M (2010) Nanoparticle stability from the nano to the meso interval. Nanoscale 2:335-342

14. Hoffmann F (1722) Observationum physico-chymicarum selectiorum. Hale, pp 375

15. Dumur F, Guerlin A, Dumas E, Bertin D, Gigmes D, Mayer CR (2011) Controlled spontaneous generation of gold nanoparticles asited by dual reducing and capping agents. Gold Bull 44:119137

16. Brust M, Walker M, Bethel D, Schiffrin DJ, Whyman R (1994) Synthesis of thiol-derivatised gold nanoparticles in a two-phase liquid-liquid system. Chem Commun 801-802

17. Liz-Marzán LM (2013) Gold-nanoparticles research before and after Brust-Schiffrin method. Chem Commun 49:16-18

18. Corma A, Concepcion P, Boronat M, Sabater MJ, Navas J, Yacaman MJ, Larios E, Posadas A, Lopez-Quintela MA, Buceta D, Mendoza E, Guilera G, Mayoral A (2013) Exceptional oxidation activity with size-controlled supported gold clusters of low atomicity. Nat Chem 5: 775-781 